\title{
ON MEROMORPHIC FUNCTIONS CONTINUOUS ON THE STOÏLOW BOUNDARY
}

\author{
PENTTI JÄRVI
}

\section{Introduction}

In this paper we study meromorphic functions on an open Riemann surface which extend continuously to the Stoillow ideal boundary ( $M C$-functions). Our main concern is how to classify the boundary elements into "essential" and "inessential" points from the point of view of $M C$-functions. It goes without saying that for most boundary elements the problem is banal: they are simply too "large" to tolerate nonconstant $M C$-functions nearby. For example, all boundary elements of positive harmonic measure are out of the question [16, p. 265]. To exclude trivialities, we confine ourselves to "admissible" points, i.e., to elements which have neighborhoods carrying nonconstant $M C$-functions.

Chapter 1 is devoted to topological properties of $M C$-functions. In particular, we exhibit the close relationship between the openness of extended functions and their covering properties. In Chapter 2, we propose a definition for removable and essential boundary points. It turns out that removability can be characterized as well in topological as in algebraic and analytic terms: via the openness of extended functions, via the field property of $M C$-functions and via a certain function-theoretic null-class, respectively. As an application, we give a solution to a problem proposed by Ozawa, concerning certain classification principles for Riemann surfaces [10, p. 751]. In Chapter 3 we give conditions, in terms of cluster sets attached to the ideal boundary, which guarantee continuous extension of the functions involved. As a very special case we obtain a recent result of Ishchanov [5].

We note in conclusion that some of the problems discussed in the present paper have been touched, although from a somewhat different point of view, in our earlier works [6] and [7].

doi:10.5186/aasfm.1984.0908 


\section{Topological properties of $M C$-functions}

1.1. Let $W$ be an open Riemann surface, and let $V$ be a subregion of $W$ with compact (possibly empty) relative boundary $\partial_{W} V$. Then $V$ is said to be an end of $W$. We often assume, as we may without loss of generality, that $\partial_{W} V$ consists of a finite number of piecewise analytic closed curves. The (Kerékjártó-) Stoïlow ideal boundary of $W$ is denoted by $\beta$ and the relative Stoilow boundary of $V$ (see [15, p. 366]) by $\beta_{V}$. The usual topological operations (closure $A \mapsto \bar{A}$, boundary $A \mapsto \partial A$ etc.) are to be taken with respect to the compactified space $W \cup \beta$ (or $V \cup \beta_{V}$ ). The class of analytic or meromorphic functions on $V$ is denoted by $A(V)$ or $M(V)$, respectively. The subclass of $A(V)$ (resp. $M(V)$ ) consisting of functions which have a finite (resp. finite or infinite) limit at every relative ideal boundary element is denoted by $A C(V)$ (resp. $M C(V))$. Whenever $f$ is a function of class $A C$ or $M C$, we let $f^{*}$ stand for the extension of $f$ to the (relative) ideal boundary. We say that $V$ is an admissible end if $M C(V)$ contains nonconstant functions. A boundary element $p \in \beta$ is called admissible provided there is an admissible end $V$ with $p \in \beta_{V}$.

Let $V$ be an end of $W$ with nice boundary, and suppose that $f \in A C\left(V \cup \partial_{W} V\right)$ is nonconstant. Assuming that $z \in C \backslash f\left(\partial_{W} V\right)$, the index of $z$ with respect to $f\left(\partial_{W} V\right)$ is defined to be

$$
i\left(z ; f\left(\partial_{W} V\right)\right)=(2 \pi)^{-1} \int_{\partial_{W} V} d \arg (f(p)-z)
$$

and the valence function, as usual,

$$
v_{f \mid V}(z)=\sum_{p \in V} \underset{p(p)=z}{ } n(p ; f),
$$

where $n(p ; f)$ denotes the multiplicity of $f$ at $p$. Then we have

Lemma 1. Suppose that $z \in C \backslash\left(f\left(\partial_{W} V\right) \cup f^{*}\left(\beta_{V}\right)\right)$. Then

$$
v_{f \mid V}=i\left(z ; f\left(\partial_{W} V\right)\right) .
$$

Proof. Fix $z_{0} \in \mathbf{C} \backslash\left(f\left(\partial_{W} V\right) \cup f^{*}\left(\beta_{V}\right)\right)$, and denote by $d$ the distance between $\left\{z_{0}\right\}$ and $f\left(\partial_{W} V\right) \cup f^{*}\left(\beta_{V}\right)$. For every $p \in \beta_{V}$ choose an open neighborhood $U_{p}$ such that $\partial U_{p}$ is contained in $V$ and $f^{*}\left(U_{p}\right) \subset D\left(f^{*}(p), d / 2\right)=\left\{z \in C|| z-f^{*}(p) \mid<d / 2\right\}$. From the open covering $\left\{U_{p} \mid p \in \beta_{V}\right\}$ of $\beta_{V}$ pick out a finite subcovering $\left\{U_{p_{1}}, \ldots, U_{p_{k}}\right\}$. Let $\left(V_{n}\right)$ be a relative exhaustion of $V \cup \partial_{W} V$ such that the components of $V \backslash V_{n}$ are noncompact. Since $F=f^{-1}\left(z_{0}\right) \cup\left(\cup_{i=1}^{k} \partial U_{p_{i}}\right)$ is a compact subset of $V$, there is a positive integer $n_{0}$ such that $F \subset V_{n}$ for $n \geqq n_{0}$. Given any component $C$ of $V \backslash V_{n_{0}}$, there is $i \in\{1, \ldots, k\}$ such that $C \subset U_{p_{i}}$. Let $B_{1}, \ldots, B_{m}$ be the components of $\partial_{W} V_{n_{0}} \cap V$. We infer that each $f\left(B_{i}\right)$ is contained in some $D\left(f^{*}\left(p_{j}\right), d / 2\right), j=1, \ldots, k$. Thus the winding number of $f\left(B_{i}\right)$ with respect to $z_{0}$ is 0 for each $i$. We conclude from the argument principle that

$$
v_{f \mid V}\left(z_{0}\right)=i\left(z_{0} ; f\left(\partial_{W} V\right)\right)
$$


Remark. The above result holds true even if $z \in f\left(\partial_{W} V\right) \backslash f^{*}\left(\beta_{V}\right)$ provided $i\left(z ; f\left(\partial_{W} V\right)\right)$ and $v_{f \mid V}$ are given a suitable interpretation in case $z \in f\left(\partial_{W} V\right)$ (see [12]).

1.2. Fix $p_{0} \in \beta$ and assume that $p_{0}$ has an admissible neighborhood, i.e., there is an admissible end $V$ such that $p_{0} \in \beta_{V}$. By passing to a subend and performing a preliminary linear fractional transformation, we obtain the situation where $V \cup \partial_{W} V$ carries a nonconstant $A C$-function $f$ with $f^{*}\left(p_{0}\right) \notin f\left(\partial_{W} V\right)$.

$1^{\circ}$. Assume first that $f^{*}\left(\beta_{V}\right)$ is nowhere dense in $C$. Denote by $G$ the component of $C \backslash f\left(\partial_{W} V\right)$ which contains $f^{*}\left(p_{0}\right)$. By Lemma $1, n=i\left(z ; f\left(\partial_{W} V\right)\right)>0$ for $z \in G$, and there is an open neighborhood $U \subset C$ of $f^{*}\left(p_{0}\right)$ such that for each $z \in U \backslash f^{*}\left(\beta_{V}\right) v_{f \mid V}(z)=n$. Now let $V=V_{1} \supset V_{2} \supset \ldots V_{j} \supset \ldots$ be a determining sequence of $p_{0}$. By applying the argument above for each $j$, we get a decreasing sequence of positive integers $\left(n_{j}=i\left(f^{*}\left(p_{0}\right) ; f\left(\partial_{W} V\right)\right)\right)$. The limit

$$
n\left(p_{0} ; f^{*}\right)=\lim _{j \rightarrow \infty} n_{j}>0
$$

is called the multiplicity or the local degree of $f^{*}$ at $p_{0}$ (cf. [4, p. 301]). It is clear that $n\left(p_{0} ; f^{*}\right)$ is independent of the choice of $\left(V_{j}\right)$. Also, it is obvious that

$$
v_{f^{*} \mid V}(z)=\sum_{\substack{f *(p)=z \\ p \in V \cup \beta_{V}}} n\left(p ; f^{*}\right)=i\left(z ; f\left(\partial_{W} V\right)\right)
$$

for every $z \in C \backslash f\left(\partial_{W} V\right)$ (note that the procedure given before applies to each $\left.p \in \beta_{V}\right)$.

$2^{\circ}$. Assume then that $f^{*}\left(\beta_{V^{\prime}}\right)$ has interior points for every subend $V^{\prime}$ of $V$ for which $p_{0} \in \beta_{V^{\prime}}$. Fix such a $V^{\prime}$, and let $\left(V_{n}^{\prime}\right)$ be a relative exhaustion of $V^{\prime}$. Let $F_{n}$ denote the closed set $f^{*}\left(\beta_{V^{\prime}}\right) \backslash f\left(V^{\prime} \backslash \bar{V}_{n}^{\prime}\right), n=1,2, \ldots$ By continuity, $f^{*}\left(\beta_{V^{\prime}}\right) \subset$ $\overline{f\left(V^{\prime} \backslash \bar{V}_{n}^{\prime}\right)}$, so that $F_{n}$ is a nowhere dense subset of $f^{*}\left(\beta_{V^{\prime}}\right)$ for each $n$. But clearly $f^{*}\left(\beta_{V^{\prime}}\right) \backslash \bigcup_{n=1}^{\infty} F_{n} \subset\left\{z \in C \mid v_{f \mid V}(z)=\infty\right\}$. In other words, $v_{f \mid V}$ becomes infinite in a set residual in $f^{*}\left(\beta_{V^{\prime}}\right)$. Therefore, given any neighborhood $U$ of $p_{0}$, we can find a sequence of points $\left(z_{n}\right)$ in $C$ such that $z_{n} \rightarrow f^{*}\left(p_{0}\right)$ and $f^{-1}\left(z_{n}\right) \cap U$ is infinite for each $n$. Hence there is no reason to define the local degree for $f^{*}$ at $p_{0}$ in this situation.

1.3. Let $X$ and $Y$ be topological spaces, and let $f: X \rightarrow Y$ be a continuous mapping. Then $f$ is said to be open if $f(U)$ is open in $Y$ for every open set $U$ in $X$. It is quasiopen, provided that for any $y \in f(X)$ and any open set $U$ in $X$ containing a compact component of $f^{-1}(y), y$ is an interior point of $f(U)$. Further, $f$ is light if every point-inverse $f^{-1}(y), y \in Y$, is totally disconnected, and $f$ is discrete if each pointinverse is discrete, i.e., consists of isolated points. Clearly, a mapping is open provided it is both quasiopen and light. For mappings of reasonably nice spaces - as is the case in this paper - quasiopenness can be characterized by the condition $\partial f(U) \subset$ $f(\partial U)$ for each relatively compact open set $U$ in $X$ [20, p. 112].

We are now ready to state some useful results concerning the behavior of $M C$ functions at the ideal boundary. 
Theorem 1. Let $W$ be an open Riemann surface, let $V$ be an admissible end of $W$ with nice boundary, and let $g \in M C\left(V \cup \partial_{W} V\right)$ be nonconstant. Let $f$ stand for $g \mid V$. Then the following statements are equivalent:

(1) $f^{*}: V \cup \beta_{V} \rightarrow \hat{\boldsymbol{C}}=\boldsymbol{C} \cup\{\infty\}$ is open.

(2) $f^{*}$ is quasiopen.

(3) $f^{*}\left(\beta_{V}\right)$ is nowhere dense in $\hat{\boldsymbol{C}}$.

(4) $f^{*}\left(\beta_{V}\right)$ is totally disconnected.

(5) $v_{f}$ is bounded.

(6) $v_{f}(z)$ is finite for each $z \in \hat{\boldsymbol{C}}$.

(7) $f^{*}$ is discrete.

Proof. Since $f^{*}$ is light, we immediately have $(1) \Leftrightarrow(2)$.

(3) $\Rightarrow(1)$ : Suppose $U$ is an open set in $V \cup \beta_{V}$ and $z_{0} \in f^{*}(U)$. If $\left(f^{*}\right)^{-1}\left(z_{0}\right) \cap V$ is nonempty, $z_{0}$ belongs to the interior of $f^{*}(U)$ by the openness of $f$. So assume that $z_{0}=f^{*}(p)$ for some $p \in \beta_{V} \cap U$. Then choose a subend $V^{\prime}$ of $V$ such that $p \in \beta_{V^{\prime}}$, $V^{\prime} \cup \beta_{V^{\prime}} \subset U, f^{*}(p) \notin f\left(\partial_{W} V^{\prime}\right)$ and $f^{*}\left(\bar{V}^{\prime}\right) \neq \hat{\boldsymbol{C}}$. Next, pick out a linear fractional transformation $\varphi$ such that $h=\varphi \circ\left(f \mid V^{\prime} \cup \partial_{W} V^{\prime}\right)$ is bounded. Plainly $h \in A C\left(V^{\prime} \cup \partial_{W} V^{\prime}\right)$. Lemma 1 now applies to $h$. Thus, letting $G$ denote the component of $C \backslash h\left(\partial_{W} V^{\prime}\right)$ that contains $h^{*}(p)=\varphi\left(z_{0}\right)$, we have $i\left(z ; h\left(\partial_{W} V^{\prime}\right)\right)=m>0$ for $z \in G$; further, $G \subset h^{*}\left(V^{\prime} \cup \beta_{V^{\prime}}\right)$. We infer that $z_{0}$ is an interior point of $f^{*}(U)$. It follows that $f^{*}(U)$ is open in $\hat{C}$.

$(1) \Rightarrow(4)$ : Suppose that $f^{*}$ gives an open mapping into $\hat{\boldsymbol{C}}$. Let $p_{0} \in \beta_{V}$ be arbitrary. By the compactness of $\beta_{V}$, it suffices to find a subend $V^{\prime}$ of $V$, with $p_{0} \in \beta_{V^{\prime}}$, such that $f^{*}\left(\beta_{V^{\prime}}\right)$ is totally disconnected. Therefore, we may again limit ourselves to the case that $h=\varphi \circ\left(f \mid V^{\prime} \cup \partial_{W} V^{\prime}\right)$ belongs to $A C\left(V^{\prime} \cup \partial_{W} V^{\prime}\right), \varphi$ being a linear fractional mapping. Assume now that $C$ is a component of $h^{*}\left(\beta_{V^{\prime}}\right)$. Fix a point $z_{0} \in \partial C$. Modifying $V^{\prime}$ slightly, we may assume that $z_{0} \notin h\left(\partial_{W} V^{\prime}\right)$. Let $G$ denote the component of $C \backslash h\left(\partial_{W} V^{\prime}\right)$ that contains $z_{0}$. It follows from the openness of $h^{*}$ that $i\left(z_{0} ; h\left(\partial_{W} V^{\prime}\right)\right)=m>0 \quad($ Lemma 1$)$.

Next, choose $r>0$ such that the disc $D\left(z_{0}, r\right) \subset G$, and set $B=\partial C \cap D\left(z_{0}, r\right)$. Let $z \in B$. We claim that $\left(h^{*}\right)^{-1}(z)$ contains at most $m$ points. Indeed, assuming that we can find $m+1$ points $p_{1}, \ldots, p_{m+1}$ in $\left(h^{*}\right)^{-1}(z)$, we can also find mutually disjoint open neighborhoods $U_{i}$ of $p_{i}, i=1, \ldots, m+1$. But then $\bigcap_{i=1}^{m+1} h^{*}\left(U_{i}\right) \cap G$ is an open neighborhood of $z$ and hence contains points from $C \backslash h^{*}\left(\beta_{V^{\prime}}\right)$. Given such a point $z^{\prime}$, the inverse image $h^{-1}\left(z^{\prime}\right)$ contains at least $m+1$ points (in $V^{\prime}$ ), whereas Lemma 1 gives $v_{h \mid V^{\prime}}\left(z^{\prime}\right)=m$. We are led to a contradiction.

Thus, $h^{*} \mid\left(h^{*}\right)^{-1}(B)$ is discrete. Since it also defines, as is readily seen, an open mapping onto $B$, we may apply [2, Lemma 2.1]. It follows that the topological dimension of $h^{*}\left(\beta_{V^{\prime}} \cap\left(h^{*}\right)^{-1}(B)\right)=B$ is 0 . We conclude that $C$ reduces to a singleton. This proves the implication $(1) \Rightarrow(4)$. 
Since the implication $(4) \Rightarrow(3)$ is trivial, we have now settled the equivalence of conditions (1) to (4).

$(4) \Rightarrow(5)$ : The number of the components of $\hat{C} \backslash\left(g\left(\partial_{W} V\right) \cup f^{*}\left(\beta_{V}\right)\right)$ is finite. In each of them $v_{f}$ is finite and constant. The desired conclusion now follows from the lower semicontinuity of $v_{f}$.

$(6) \Rightarrow(3)$ : Suppose, for the moment, that $f^{*}\left(\beta_{V}\right)$ has interior points. The argument of Section 2.1 then yields the result that $v_{f}$ is infinite in a residual part of $f^{*}\left(\beta_{V}\right)$. This contradicts (6).

$(4) \Rightarrow(7)$ : Since discreteness is a local property, we may again refer to the results of Section 1.2 about $A C$-functions: (7) is indeed a direct consequence of formula (A).

The implications $(5) \Rightarrow(6)$ and $(7) \Rightarrow(6)$ being trivial, the proof is complete.

Corollary 1. Let $W$ be an open Riemann surface, let $\beta$ be the ideal boundary of $W$ and suppose that $f \in M C(W)$ is nonconstant. Then either

(a) $f^{*}(\beta)$ is totally disconnected, in which case $v_{f^{*}}(z)=\sum_{f^{*}(p)=z} n\left(p ; f^{*}\right)$ is finite and constant, or

(b) the interior of $f^{*}(\beta)$ is nonempty, and the set $\left\{z \in \hat{C} \mid v_{f}(z)=\infty\right\}$ is residual in $f^{*}(\beta)$.

The next result, a direct consequence of Lemma 1, was given and utilized in [6].

Corollary 2. Let $W$ and $\beta$ be as above, and let $f \in A C(W)$. Then $f^{*}(\beta)=$ $f^{*}(W \cup \beta)$.

The following corollary provides a generalization of Stoïlow's uniqueness theorem $[17$, p. 124].

Corollary 3. Let $W$ be an open Riemann surface, and let $V$ be an end of $W$ such that the set $\beta_{V}$ is infinite. Suppose that $f \in M C(V)$ and $f^{*}(p)=0$ for every $p \in \beta_{V}$. Then $f$ vanishes identically on $V$.

To give an example of nontrivial $A C$-functions, take a compact totally disconnected set $E \subset C$ such that $m(E)$, the two-dimensional Lebesgue measure of $E$, is positive and set

$$
f(z)=\iint_{E} \frac{1}{\zeta-z} d m_{\zeta}
$$

It turns out that $f$ belongs to $A C(\hat{\boldsymbol{C}} \backslash E)$; for details see e.g. [3, p. 79-80].

Remark. We point out that all the results given in this chapter are of a purely topological character. In particular, they remain valid if the analyticity of mappings is replaced by interiority (in the sense of Stoillow). Of course, the requirement that boundary elements be admissible can then be dropped. 


\section{Essential and removable boundary elements}

2.1. We begin with some terminology. Let $E$ be a proper closed subset of $\hat{\boldsymbol{C}}$. Then $E$ is said to be of class $N_{C}$ if, for each domain $G \subset \hat{\boldsymbol{C}}$ with $E \subset G$, every function $G \rightarrow C$ continuous on $G$ and analytic on $G \backslash E$ is actually analytic all over $G$. The subclass of $N_{C}$ constituted by the totally disconnected elements of $N_{C}$ is denoted by $N_{C}^{\prime}$. It is known that every closed set $E \subset \hat{C}$ of $\sigma$-finite linear mesure is of class $N_{C}$ and, on the other hand, no set whose Hausdorff dimension exceeds 1 is of class $N_{C}$ (see e.g. [3]). In the natural way (see [6, p. 308]), the classes $N_{C}$ and $N_{C}^{\prime}$ can be generalized for arbitrary Riemann surfaces.

Let $V$ denote an end of an open Riemann surface $W$. We say that $V$ satisfies the absolute $A C$-maximum principle if for each subend $V^{\prime}$ of $V$ and for each $f \in A C\left(V^{\prime} \cup\right.$ $\left.\partial_{W} V^{\prime}\right)$

$$
\sup \left\{|f(p)| \mid p \in V^{\prime} \cup \partial_{W} V^{\prime}\right\}=\max \left\{|f(p)| \mid p \in \partial_{W} V^{\prime}\right\} .
$$

Theorem 2. Let $W$ be an open Riemann surface, let $\beta$ be the ideal boundary of $W$, and let $p \in \beta$ be an admissible boundary point. Then the following properties are equivalent:

(1) There is an end $V \subset W$ with $p \in \beta_{V}$ such that for every subend $V^{\prime}$ of $V$ and for every nonconstant $f \in M C\left(V^{\prime}\right), f^{*}$ defines an open mapping $V^{\prime} \cup \beta_{V^{\prime}} \rightarrow \hat{C}$.

(2) There is an end $V \subset W$ with $p \in \beta_{V}$ which satisfies the absolute $A C$-maximum principle.

(3) There is an end $V \subset W$ with $p \in \beta_{V}$ such that for every subend $V^{\prime}$ of $V$, $M C\left(V^{\prime}\right)$ constitutes a field.

(4) There is an end $V \subset W$ with $p \in \beta_{V}$ such that for every subend $V^{\prime}$ of $V$ and for every $f \in M C\left(V^{\prime}\right), f^{*}\left(\beta_{V^{\prime}}\right)$ belongs to $N_{C}^{\prime}$.

(5) There is an end $V \subset W$ with $p \in \beta_{V}$ and a nonconstant function $f$ in $M C(V)$ such that $f^{*}\left(\beta_{V}\right)$ belongs to $N_{C}^{\prime}$.

Proof. (1) $\Rightarrow(2)$ : Suppose that $V \subset W$ fulfils the hypotheses of (1). Let $V^{\prime}$ be a subend of $V$, and let $f \in A C\left(V^{\prime} \cup \partial_{W} V^{\prime}\right)$ be nonconstant. Since $V^{\prime} \cup \beta_{V^{\prime}}$ is an open set in $V \cup \beta_{V}, f^{*}\left(V^{\prime} \cup \beta_{V^{\prime}}\right)$ is open in $C$. Therefore $f$ attains its maximum at a point on $\partial_{W} V^{\prime}$.

(2) $\Rightarrow(1):$ Suppose $V \subset W$ sátisfies the absolute $A C$-maximum principle, and for some subend $V^{\prime} \subset V$ and some nonconstant $f \in M C\left(V^{\prime}\right) f^{*}$ fails to be open. By the equivalence $(1) \Leftrightarrow(2)$ in Theorem 1 , we can find a relatively compact open set $U \subset V^{\prime} \cup \beta_{V^{\prime}}$ such that $\partial f^{*}(U) \leftarrow f^{*}(\partial U)$. Pick out a point $z_{0} \in \partial f^{*}(U) \backslash f^{*}(\partial U)$. It is clear that $z_{0}=f^{*}\left(p_{0}\right)$ for some $p_{0} \in U \cap \beta_{V^{\prime}}$. Now choose an end $V^{\prime \prime} \subset U$ such that $p_{0} \in \beta_{V^{\prime \prime}}$. Plainly, $z_{0} \notin f\left(\partial_{W} V^{\prime \prime}\right)$. Let $z_{1}$ stand for a point in $C \backslash f^{*}\left(\bar{V}^{\prime \prime}\right)$ such that $\left|z_{1}-z_{0}\right|<\min \left\{\left|z_{1}-z\right| \mid z \in f\left(\partial_{W} V^{\prime \prime}\right)\right\}$ (we may assume that $f^{*}\left(\bar{V}^{\prime \prime}\right)$ lies in $C$ ). Denote by $g$ the function $p \mapsto\left(z_{1}-f(p)\right)^{-1}, p \in V^{\prime \prime} \cup \partial_{W} V^{\prime \prime}$. It is clear that 
$g \in A C\left(V^{\prime \prime} \cup \partial_{W} V^{\prime \prime}\right)$ and $\left|g^{*}\left(p_{0}\right)\right|>\max \left\{|g(p)| \mid p \in \partial_{W} V^{\prime \prime}\right\}$. We have obtained the desired contradiction.

$(1) \Rightarrow(5)$ : Let $V$ be an admissible end with $p \in \beta_{V}$, and let $f \in M C\left(V \cup \partial_{W} V\right)$ be nonconstant. Reducing $V$ and performing a preliminary linear fractional transformation, we may assume that $f$ belongs to $A C\left(V \cup \partial_{W} V\right)$. Since $f^{*}$ gives an open mapping of $V \cup \beta_{V}$ into $C$, it follows from Theorem 1 that $f^{*}\left(\beta_{V}\right)$ is totally disconnected. Thus we may arrange $f^{*}\left(\beta_{V}\right) \cap f\left(\partial_{W} V\right)=\emptyset$.

Let $n$ stand for $\max \left\{i\left(z ; f\left(\partial_{W} V\right)\right) \mid z \in C \backslash f\left(\partial_{W} V\right)\right\}$. Then $v_{f \mid V}(z)$ is bounded by $n$, in view of formula (A) in Section 1.2. Let $E_{i}$ denote $\left\{z \in f^{*}\left(\beta_{V}\right) \mid v_{f \mid V}(z) \leqq i\right\}$, $i=0, \ldots, n-1$; then $E_{n-1}=f^{*}\left(\beta_{V}\right)$. Since $f$ is open, each $E_{i}$ is closed. Moreover, we claim that each $E_{i}$ belongs to $N_{C}^{\prime}$.

Assume that $E_{0}$ is not of class $N_{C}^{\prime}$. By a standard application of Cauchy's integral formula (see e.g. [6, Lemma 4]), we can find a nonconstant function $g$ in $A C\left(\hat{C} \backslash E_{0}\right)$. Clearly $g \circ f$ belongs to $A C(V)$. Since $g^{*}\left(E_{0}\right) \subset(g \circ f)^{*}\left(\beta_{V}\right)$, and $g^{*}\left(E_{0}\right)$ contains interior points (see Corollary 2 to Theorem 1 ), we conclude by Theorem 1 that $(g \circ f)^{*}$ is not open. This contradicts (1). Suppose next that, for some $i$, $E_{i}$ is of class $N_{C}^{\prime}$, and fix a point $z_{0} \in E_{i+1} \backslash E_{i}$. Choose a neighborhood $U$ of $z_{0}$ such that $\partial U$ is an analytic Jordan curve with $\partial U \cap\left(f^{*}\left(\beta_{V}\right) \cup f\left(\partial_{W} V\right)\right)=\emptyset$ and $f^{-1}(U)$ contains $j(j \leqq i+1)$ relatively compact mutually disjoint Jordan regions $V_{k}$ in $V$ such that each $z \in U$ has exactly $i+1$ antecedents in $\bigcup_{k=1}^{j} V_{k}$ (with due account of multiplicities). Then $f \mid V \backslash \bigcup_{k=1}^{j} V_{k}$ assumes no value in $E_{i+1} \cap U$. Now $E_{i+1} \cap U$ must be of class $N_{C}^{\prime}$, for otherwise - reproducing the argument given above - we would again arrive at a contradiction with (1). Since $z_{0}$ was arbitrary, and belonging to $N_{C}^{\prime}$ is a local property (see e.g. [6, p. 308]), we infer $E_{i+1} \in N_{C}^{\prime}$. It follows that $E_{n-1}=f^{*}\left(\beta_{V}\right)$ is of class $N_{C}^{\prime}$.

$(5) \Rightarrow(4)$ : Suppose $V \subset W$ is an end which carries a nonconstant $M C$-function $f_{0}$ with $f_{0}^{*}\left(\beta_{V}\right) \in N_{C}^{\prime}$. Let $V^{\prime}$ be a subend of $V$. Modifying $V^{\prime}$ slightly we obtain $f_{0}\left(\partial_{W} V^{\prime}\right) \cap f_{0}^{*}\left(\beta_{V^{\prime}}\right)=\emptyset$. Let $G$ be a component of $\hat{C} \backslash f_{0}\left(\partial_{W} V^{\prime}\right)$ such that $f_{0}^{*}\left(\beta_{V^{\prime}}\right) \cap$ $G \neq \emptyset$. Then $v_{f_{0} \mid V^{\prime}}$ is finite and constant, say $n$, in $G \backslash f_{0}^{*}\left(\beta_{V^{\prime}}\right)$. Assume that $f \in M C\left(V^{\prime}\right)$ is nonconstant. By an argument familiar from the context of compact Riemann surfaces, it can be shown that $f$ satisfies on $f_{0}^{-1}\left(G \backslash f_{0}^{*}\left(\beta_{V^{\prime}}\right)\right)$ an identity

$$
f^{n}+\sum_{i=1}^{n}\left(a_{i} \circ f_{0}\right) f^{n-i}=0,
$$

where $a_{1}, \ldots, a_{n}$ are meromorphic functions on $G \backslash f_{0}^{*}\left(\beta_{V^{\prime}}\right)$. Arguing as in [6, p. $309]$, it can be shown that for each $i, a_{i}$ admits a meromorphic extension over $f_{0}^{*}\left(\beta_{V^{\prime}}\right) \cap G$ (this is the point where use is made of the assumption $f_{0}^{*}\left(\beta_{V}\right) \in N_{C}^{\prime}$ ). Henceforth we regard each $a_{i}$ as defined and meromorphic all over $G$.

Denote by $\widetilde{G}$ the Riemann surface of the relation

$$
P(z, w)=w^{n}+\sum_{i=1}^{n} a_{i}(z) w^{n-i}=0, \quad z \in G,
$$

i.e., the totality of pairs $\left(z, w_{z}\right)$, where $z \in G$ and $w_{z}$ is a function element with center $z$ and associated with the equation $P(z, w)=0$. Note that $\widetilde{G}$ is a finite union of con- 
nected Riemann surfaces. The functions $c:\left(z, w_{z}\right) \mapsto z$ and $v:\left(z, w_{z}\right) \mapsto w_{z}(z)$ are meromorphic on $\widetilde{G}$. Obviously, $f^{*}\left(\left(f_{0}^{*}\right)^{-1}\left(f_{0}^{*}\left(\beta_{V^{\prime}}\right) \cap G\right) \cap \beta_{V^{\prime}}\right) \subset v\left(c^{-1}\left(f_{0}^{*}\left(\beta_{V^{\prime}}\right) \cap G\right)\right)$. Hence by [6, Lemma 2], $f^{*}\left(\left(f_{0}^{*}\right)^{-1}\left(f_{0}^{*}\left(\beta_{V^{\prime}}\right) \cap G\right) \cap \beta_{V^{\prime}}\right)$ is of class $N_{C}^{\prime}$. Being a finite union of sets of this kind, $f^{*}\left(\beta_{V^{\prime}}\right)$ also belongs to $N_{C}^{\prime}$.

$(5) \Rightarrow(3)$ : Suppose $V$ and $f \in M C(V)$ satisfy (5). Fix $p \in \beta_{V}$. As in [6, Theorem 8], there is a subend $V^{\prime} \subset V$ with $p \in \beta_{V^{\prime}}$ and an analytic function $f_{0} \in A C\left(V^{\prime}\right): V^{\prime} \rightarrow D=$ $\{z \in C|| z \mid<1\}$ such that, given any $g \in M C\left(V^{\prime}\right)$, one can find a unique $h \in M(D)$ satisfying $g=h \circ f_{0}$. Making use of this composition, we can readily obtain the conclusion.

(3) $\Rightarrow(2)$ : Suppose there is a subend $V^{\prime}$ of $V$ and a function $f \in A C\left(V^{\prime} \cup \partial_{W} V^{\prime}\right)$ with $\max \left\{|f(p)| \mid p \in \partial_{W} V^{\prime}\right\}<\max \left\{\left|f^{*}(p)\right| \mid p \in \bar{V}^{\prime}\right\}=r$. Pick out a point $p_{0} \in \beta_{V^{\prime}}$ such that $\left|f^{*}\left(p_{0}\right)\right|=r$. Let $\varphi$ be a conformal mapping of the disc $D(0, r)$ onto the half-strip $\{z \in C|\operatorname{Re} z<0,| \operatorname{Im} z \mid<1\}$ such that $f^{*}\left(p_{0}\right)$ corresponds to the point $-\infty$. It is clear that the functions $h=\exp (\varphi \circ f)$ and $g=\exp ((1-i) \cdot \varphi \circ f)$ belong to $A C\left(V^{\prime} \cup \partial_{W} V^{\prime}\right)$ (exp stands for $\left.z \mapsto e^{z}\right)$; moreover, $h(p)$ and $g(p) \rightarrow 0$ as $p \rightarrow p_{0}$ in $V^{\prime}$. Now choose a sequence of points $\left(p_{n}\right)$ in $V^{\prime}$ such that $p_{n} \rightarrow p_{0}$ and $\operatorname{Re}\left((\varphi \circ f)\left(p_{n}\right)\right)=-n$ for large $n$. Then $\left|h\left(p_{n}\right)\right|=\exp (-n)$ and $\left|g\left(p_{n}\right)\right|=$ $\exp \left(-n+\operatorname{Im}\left((\varphi \circ f)\left(p_{n}\right)\right)\right)$. Hence $\left|g\left(p_{n}\right) / h\left(p_{n}\right)\right|=\exp \left(\operatorname{Im}\left((\varphi \circ f)\left(p_{n}\right)\right)\right)$, whence $\exp (-1) \leqq\left|g\left(p_{n}\right) / h\left(p_{n}\right)\right| \leqq \exp (1)$ for large $n$. We conclude that

$$
\lim _{\substack{p \rightarrow p_{0} \\ p \in V^{\prime}}}(g / h)(p) \neq 0, \infty .
$$

Similarly, a simple calculation yields $\arg g\left(p_{n}\right)=\operatorname{Im}\left((\varphi \circ f)\left(p_{n}\right)\right)-\operatorname{Re}\left((\varphi \circ f)\left(p_{n}\right)\right)$ and $\arg h\left(p_{n}\right)=\operatorname{Im}\left((\varphi \circ f)\left(p_{n}\right)\right)$. Thus, $\arg h\left(p_{n}\right)$ remains bounded, while $\arg g\left(p_{n}\right)$ varies unboundedly as $n \rightarrow \infty$. Hence $\arg (g / h)\left(p_{n}\right)$ also varies unboundedly as $n \rightarrow \infty$. But this state of affairs is in apparent contradiction with (*). The implication follows.

The remaining implication (4) $\Rightarrow(1)$ follows immediately from Theorem 1.

In view of the preceding theorem, it seems reasonable to make the following definition (cf. [7, p. 320]):

Definition. Let $p \in \beta$ be an admissible boundary element. Then $p$ is said to be (AC-)removable if there is an end $V$ with $p \in \beta_{V}$ and a nonconstant function $f \in M C(V)$ such that $f^{*}\left(\beta_{V}\right)$ is of class $N_{C}^{\prime}$. Otherwise $p$ is called essential. A closed subset $\beta^{\prime}$ of $\beta$ is said to be removable if each element of $\beta^{\prime}$ is removable.

It is clear that the removable boundary points constitute a relatively open subset of $\beta$.

Suppose $W$ is parabolic. Then every admissible boundary point is removable; indeed, given any end $V \subset W$ and any $f \in M C(V), f^{*}\left(\beta_{V}\right)$ is of logarithmic capacity zero (see [9]). More generally, the same is true of Riemann surfaces satisfying the absolute $A B$-maximum principle ([12], [10], [6]); this case can be characterized by the relation $f^{*}\left(\beta_{V}\right) \in N_{B}\left[6\right.$, p. 304] (for $N_{B}$ and other standard null-classes see [1] or [15, 
Chapter II]). It is to be noted that there are even parabolic surfaces which entirely lack admissible boundary elements [4, p. 298].

2.2. Our next theorem describes the class of globally defined $M C$-functions in case $\beta$ is removable.

Theorem 3. Let $W$ be an open Riemann surface, and suppose that the ideal boundary $\beta$ of $W$ is removable. Then either

(a) $M C(W)=C$, or

(b) $M C(W)$ is a field algebraically isomorphic to the field of rational functions on a compact Riemann surface $W^{\prime}$, which is uniquely determined up to a conformal equivalence. Moreover, the isomorphism is induced by an analytic mapping of $W$ into $W^{\prime}$.

Proof. Suppose that $M C(W)$ contains a nonconstant function $f$. By definition and by Theorem 2, each boundary element $p \in \beta$ has a neighborhood $U_{p}$ with $\partial U_{p}$ in $W$ such that $f^{*}\left(\beta \cap U_{p}\right)$ is of class $N_{C}^{\prime}$. By compactness, we can pick out $U_{p_{1}}, \ldots, U_{p_{n}}$ such that $\beta \subset \bigcup_{i=1}^{n} U_{p_{i}}$. Hence $f^{*}(\beta) \subset \bigcup_{i=1}^{n} f^{*}\left(\beta \cap U_{p_{i}}\right)$, whence $f^{*}(\beta)$ is of class $N_{C}^{\prime}$. The theorem now follows from [6, Theorem 6].

A local counterpart to the preceding theorem is

Theorem 4. Let $W$ be an open Riemann surface with ideal boundary $\beta$, and suppose that $p \in \beta$ is removable. Then there is an end $V$ of $W$ with $p \in \beta_{V}$ and an $A C$ function $f_{0}: V \rightarrow D=\{z \in C|| z \mid<1\}$ such that, given any $f \in M C(V)$, one can find $a$ unique $g \in M(D)$ (=the class of meromorphic functions on $D$ ) satisfying $f=g \circ f_{0}$. Accordingly, $M C(V)$ is isomorphic to the field $M(D)$.

Proof. See [6, Theorem 8].

Suppose next that an end $V \subset W$ has finite genus. Then $V$ can be imbedded conformally in a compact Riemann surface $U^{*}$. Therefore $\beta_{V}$ can be realized as a subset of $U^{*}$. Thus, it makes sense to ask what $\beta_{V}$ looks like near a removable boundary point. An answer is given by

Theorem 5. Let $W$ be an open Riemann surface, and let $V \subset W$ be an end of finite genus. Suppose that $\partial_{W} V$ is a finite union of analytic Jordan curves and $\beta_{V}$ is removable. Then there exists a finite Riemann surface $V^{*}$ and a compact subset $E \subset V^{*}$ of class $N_{C}^{\prime}$ such that $V$ is conformally equivalent to $V^{*} \backslash E$. Further, $V^{*}$ is uniquely determined up to a conformal equivalence.

Proof. Let $p \in \beta_{V}$, and choose a planar end $V^{\prime} \subset V$ such that $p \in \beta_{V^{\prime}}, \partial_{W} V^{\prime}$ is a Jordan curve, and $M C\left(V^{\prime}\right)$ contains a nonconstant function $f$. Assume also that $V^{\prime}$ satisfies condition (4) in Theorem 2. By [11, Theorem 3], we can find a Jordan domain $G \subset C$, a compact totally disconnected set $F \subset G$ and a sense-preserving homeomorphism $\varphi: V^{\prime} \rightarrow G \backslash F$. Further, $\beta_{V^{\prime}}$ and $F$ being totally disconnected, $\varphi$ admits a homeomorphic extension $\varphi^{*}: V^{\prime} \cup \beta_{V^{\prime}} \rightarrow G$. Consider the continuous 
mapping $g=f^{*} \circ\left(\varphi^{*}\right)^{-1}: G \rightarrow \hat{\boldsymbol{C}}$. It is clear that $g$ is light. Also, $g \mid G \backslash F$ is open and sense-preserving, and $g(F)=f^{*}\left(\beta_{V^{\prime}}\right)$ is totally disconnected (of class $N_{C}^{\prime}$ in fact). Hence by [18, Theorem 9], $g$ is light and open on $G$. By Stoïlow's theorem [17, p. 121], there is a plane domain $G^{\prime}$, a sense-preserving homeomorphism $\psi: G \rightarrow G^{\prime}$ and a meromorphic function $h$ on $G^{\prime}$ such that $g=h \circ \psi$. As in [7, p. 319], we see that $\psi \circ \varphi$ defines a conformal mapping $V^{\prime} \rightarrow G^{\prime} \backslash \psi(F)$. Further, by condition (4) in Theorem $2\left(\psi \circ \varphi^{*}\right)\left(\beta_{V^{\prime}}\right)$ is of class $N_{C}^{\prime}$.

Altogether, for each $p \in \beta_{V}$ there is an open neighborhood $U_{p} \subset V \cup \beta_{V}$ of $p$ and a homeomorphism $\Phi_{p}$ of $U_{p}$ onto a plane domain such that $\Phi_{p} \mid U_{p} \backslash \beta_{V}$ is conformal and $\Phi_{p}\left(\beta_{V} \cap U_{p}\right)$ is of class $N_{C}^{\prime}$. The very definition of $N_{C}^{\prime}$ implies that the transition mappings $\Phi_{p} \circ \Phi_{q}^{-1}: \Phi_{q}\left(U_{p} \cap U_{q}\right) \rightarrow \Phi_{p}\left(U_{p} \cap U_{q}\right)$ are actually conformal. Accordingly, $V \cup \beta_{V}$ can be given a conformal structure, compatible with that of $V$, which makes $V \cup \beta_{V}$ a finite Riemann surface. Clearly, $\beta_{V}$ is of class $N_{C}^{\prime}$ in $V^{*}=$ $V \cup \beta_{V}$ (see [6, p. 308]). Hence we may set $E=\beta_{V}$; the inclusion mapping $i: V \rightarrow V^{*}$ defines the desired conformal homeomorphism $V \rightarrow V^{*} \backslash E$.

To prove the uniqueness, suppose that the pairs $\left(V_{1}^{*}, E_{1}\right)$ and $\left(V_{2}^{*}, E_{2}\right)$ have the required properties. Let $\varphi_{1}: V \rightarrow V_{1}^{*} \backslash E_{1}$ and $\varphi_{2}: V \rightarrow V_{2}^{*} \backslash E_{2}$ denote the related conformal homeomorphisms. Then $\varphi=\varphi_{2} \circ \varphi_{1}^{-1}$ maps $V_{1}^{*} \backslash E_{1}$ conformally onto $V_{2}^{*} \backslash E_{2}$. Since $E_{1}$ and $E_{2}$ are totally disconnected, $\varphi$ admits a homeomorphic extension $\varphi^{*}: V_{1}^{*} \rightarrow V_{2}^{*}$. Finally, $E_{1}$ being of class $N_{C}^{\prime}$ in $V_{1}^{*}, \varphi^{*}$ is conformal throughout $V_{1}^{*}$.

Remark 1. As appears from the proof, the uniqueness of $V^{*}$ follows already from the requirement that the set $E$, the realization of the ideal boundary, be totally disconnected. It should be noted that there are realizations of removable boundaries which contain proper continua. This state of affairs derives from the fact that there are sets of class $N_{C}^{\prime}$ which do not belong to $N_{S B}$.

Remark 2. Suppose $f \in M C(V)$ under the hypotheses of the preceding theorem, and let $\varphi$ map $V$ conformally onto $V^{*} \backslash E$ with $E$ in $N_{C}^{\prime}$. Then $f \circ \varphi^{-1} \in$ $M C\left(V^{*} \backslash E\right)$ and, since $E \in N_{C}^{\prime},\left(f \circ \varphi^{-1}\right)^{*}=f^{*} \circ\left(\varphi^{-1}\right)^{*}$ is meromorphic in $V^{*}$. In this sense, $f$ can be continued to be "meromorphic" on the ideal boundary.

Our next theorem gives a criterion to recognize the situation described above.

Theorem 6. Let $W$ be an open Riemann surface, and let $V \subset W$ be an end whose relative boundary $\partial_{W} V$ consists of a finite number of closed analytic curves. Suppose $A C\left(V \cup \partial_{W} V\right)$ separates the points of $V \cup \partial_{W} V$, and for each $f \in A C\left(V \cup \partial_{W} V\right)$ and for each subend $V^{\prime}$ of $V$

$$
\max \left\{|f(p)| \mid p \in \partial_{W} V^{\prime}\right\}=\sup \left\{|f(p)| \mid p \in V^{\prime} \cup \partial_{W} V^{\prime}\right\} .
$$

Then there exists a finite Riemann surface $V^{*}$ and a compact subset $E \subset V^{*}$ of class $N_{C}^{\prime}$ such that $V$ is conformally equivalent to $V^{*} \backslash E ; V^{*}$ is uniquely determined up to a conformal equivalence. 
Proof. Since $A C\left(V \cup \partial_{W} V\right)$ is point-separating, and each $f \in A C\left(V \cup \partial_{W} V\right)$ attains its maximum on $\partial_{W} V$, it follows from a theorem of Royden [13, Theorem 3] that $V$ has finite genus.

Let $f \in A C\left(V \cup \partial_{W} V\right)$, and let $K \subset V \cup \beta_{V}$ be a compact set. By assumption and by the total disconnectedness of $\beta_{V}$, it is readily seen that $\left|f^{*}\left(p_{0}\right)\right| \leqq$ $\max \left\{\mid f^{*}(p) \| p \in \partial K\right\}$ for each $p_{0} \in K$. Therefore, taken as an algebra of functions defined in $V \cup \beta_{V}, A C\left(V \cup \partial_{W} V\right)$ constitutes a maximum modulus algebra in the sense of [8]. Hence by [8, Theorem 1], every $f \in A C\left(V \cup \partial_{W} V\right)$ defines a quasiopen mapping $f^{*}: V \cup \beta_{V} \rightarrow C$. By Theorem $1, f^{*}\left(\beta_{V}\right)$ is totally disconnected.

Fix a nonconstant $f \in A C\left(V \cup \partial_{W} V\right)$. Combining [11, Theorem 3], [18, Theorem 9] and Stoillow's theorem as in the proof of the preceding theorem, we infer that there exist a finite Riemann surface $V^{*}$, a compact totally disconnected set $E \subset V^{*}$ and a conformal homeomorphism $\varphi: V \rightarrow V^{*} \backslash E$. Of course, $V^{*}$ can be taken as a subregion of a compact Riemann surface $\tilde{V}$. Suppose $E$ fails to be of class $N_{C}^{\prime}$ in $V^{*}$ (and in $\tilde{V})$. By [6, Lemma 4], we can then find a nonconstant function $g$ in $A C(\tilde{V} \backslash E)$. Further, by Corollary 2 to Theorem 1, $g^{*}\left(V^{*}\right) \subset g^{*}(\tilde{V})=g^{*}(E)=(g \circ \varphi)^{*}\left(\beta_{V}\right)$. Hence $g \circ \varphi$, albeit a member of $A C\left(V \cup \partial_{W} V\right)$, by the reflection principle, does not attain its maximum on $\partial_{W} V$. This contradicts (1).

The uniqueness of $V^{*}$ is proven as in the preceding theorem.

Remark. It seems possible that Theorem 6 remains valid even if condition (1) is imposed only on $V$. Actually, a result of this sort holds for the algebra of bounded analytic functions on $V$, as shown by Wermer [19] and Royden [13].

2.3. A Riemann surface $W$ is said to satisfy the absolute $A B$-maximum principle, briefly $W \in \mathscr{M}_{B}$, if

$$
\sup \left\{|f(p)| \mid p \in V \cup \partial_{W} V\right\}=\max \left\{|f(p)| \mid p \in \partial_{W} V\right\}
$$

for every end $V \subset W$ and for every $f \in A B\left(V \cup \partial_{W} V\right.$ ) (=the class of bounded analytic functions on $V \cup \partial_{W} V$ ) (see [12], [10]). Further, $W$ is said to belong to the class $\mathscr{D}_{B}$ if, for every end $V \subset W$, the cluster set $\mathrm{Cl}\left(f ; \beta_{V}\right)$ of every $f \in A B\left(V \cup \partial_{W} V\right)$ attached to $\beta_{V}$ is totally disconnected [10]. Finally, $W$ is said to belong to the class $\mathscr{A}_{B}$ provided that $A B\left(V \cup \partial_{W} V\right) \subset A C\left(V \cup \partial_{W} V\right)$ for every end $V \subset W[10]$. As to the inclusion relations between these classes, it is immediate that $\mathscr{D}_{B} \subset \mathscr{A}_{B}$; further, the work of Royden [12] readily brings in the equality $\mathscr{M}_{B}=\mathscr{D}_{B}$ (see [6]). In the next theorem, it will be shown that $\mathscr{A}_{B}=\mathscr{D}_{B}$ also. This settles a problem proposed by Ozawa [10, p. 751].

Theorem 7. The three classes defined above coincide:

$$
\mathscr{M}_{B}=\mathscr{D}_{B}=\mathscr{A}_{B}
$$

Proof. It remains to prove that $\mathscr{A}_{B} \subset \mathscr{D}_{B}$. Assume the contrary, and let $W$ be a Riemann surface in $\mathscr{A}_{B} \backslash \mathscr{D}_{B}$. By definition, there exist an end $V \subset W$ and a function $f \in A B\left(V \cup \partial_{W} V\right)=A C\left(V \cup \partial_{W} V\right)$ such that $f^{*}\left(\beta_{V}\right)$ contains proper continua. 
Hence by Theorem 1, $f^{*} \mid V \cup \beta_{V}$ is not open. By Theorem 2, we can then find a subend $V^{\prime}$ of $V$ and a function $g$ in $A C\left(V^{\prime} \cup \partial_{W} V^{\prime}\right)$ such that $\max \left\{\mid g(p) \| p \in \partial_{W} V^{\prime}\right\}<$ $r=\max \left\{\left|g^{*}(p)\right| \mid p \in \bar{V}^{\prime}\right\}=\max \left\{\left|g^{*}(p)\right| \mid p \in \beta_{V^{\prime}}\right\}$. Pick out a point $p_{0} \in \beta_{V^{\prime}}$ such that $\left|g^{*}\left(p_{0}\right)\right|=r$.

Let $\varphi$ be a conformal mapping of the disc $D(0, r)$ onto the strip domain bounded by the lines $\operatorname{Re} z=0$ and $\operatorname{Re} z=1$ such that $g^{*}\left(p_{0}\right)$ corresponds to the point $+\infty i$. Clearly, $\exp (\varphi \circ g)$ belongs to $A B\left(V^{\prime} \cup \partial_{W} V^{\prime}\right)$. Yet $\exp (\varphi \circ g)$ fails to have a limit as $p \rightarrow p_{0}$ in $V^{\prime}$. This contradiction completes the proof.

Corollary. Let $W$ be an open Riemann surface. Then $W$ satisfies the absolute $A B$-maximum principle if and only if $A B\left(V \cup \partial_{W} V\right)=A C\left(V \cup \partial_{W} V\right)$ for every end $V \subset W$.

Remark. In case $W$ is planar or, more generally, has finite genus, the equality $\mathscr{A}_{B}=\mathscr{M}_{B}$ follows immediately from a result of Rudin. In fact, whenever $p \in \partial W$ is an essential boundary point in the sense of Rudin [14, p. 333], there exists an analytic function $f$ in $A B(W)$, bounded by 1 , such that $\mathrm{Cl}(f ; p)$, the cluster set of $f$ at $p$, equals $\overline{D(0,1)}$ [14, Theorem 14].

\section{A condition for continuity}

3.1. Let $V$ be an end of an open Riemann surface $W$, and let $f$ be a nonconstant bounded analytic function on $V$. Suppose that no $\mathrm{Cl}(f ; p), p \in \beta_{V}$, separates the plane (note that each $\mathrm{Cl}(f ; p)$ is connected) and $\mathrm{Cl}\left(f ; \beta_{V}\right)=\cup_{p \in \beta_{V}} \mathrm{Cl}(f ; p)$ is nowhere dense in $C$. We say that $p \in \beta_{V}$ is a generalized antecedent of a point $z \in C$ with respect to $f$ provided there is a sequence of points $\left(p_{n}\right)$ in $V$ such that $p_{n} \rightarrow p$ and $f\left(p_{n}\right) \rightarrow z$ as $n \rightarrow \infty$; in other words, $p$ is a generalized antecedent of $z$ if and only if $z \in \mathrm{Cl}(f ; p)$.

Let $V^{\prime}$ be a subend of $V$ such that $\partial_{W} V^{\prime}$ is contained in $V$ and consists of a finite number of piecewise analytic closed curves. We will need the following generalization of Lemma 1.

Lemma 2. Suppose that $z \in C \backslash\left(f\left(\partial_{W} V^{\prime}\right) \cup \mathrm{Cl}\left(f ; \beta_{V^{\prime}}\right)\right)$. Then

$$
v_{f \mid V^{\prime}}(z)=i\left(z ; f\left(\partial_{W} V^{\prime}\right)\right) .
$$

Proof. Fix $z_{0} \in C \backslash\left(f\left(\partial_{W} V^{\prime}\right) \cup \mathrm{Cl}\left(f ; \beta_{V^{\prime}}\right)\right)$, and denote by $d$ the distance between $\left\{z_{0}\right\}$ and $f\left(\partial_{W} V^{\prime}\right) \cup \mathrm{Cl}\left(f ; \beta_{V^{\prime}}\right)$. For every $p \in \beta_{V^{\prime}}$ choose an open neighborhood $U_{p}$ such that $\partial U_{p} \subset V^{\prime}$ and $z_{0}$ can be joined to $\infty$ by an arc in $C \backslash \overline{f\left(U_{p} \cap V^{\prime}\right)}$; this is possible because $C \backslash \mathrm{Cl}(f ; p)$ is assumed to be connected. From the open covering $\left\{U_{p} \mid p \in \beta_{V^{\prime}}\right\}$ of $\beta_{V^{\prime}}$ pick out a finite subcovering $\left\{U_{p_{1}}, \ldots, U_{p_{k}}\right\}$. Let $\left(V_{n}^{\prime}\right)$ be a relative exhaustion of $V^{\prime} \cup \partial_{W} V^{\prime}$ such that the components of $V^{\prime} \backslash V_{n}^{\prime}$ are noncompact. Since $F=\bigcup_{i=1}^{k} \partial U_{p_{i}}$ is a compact subset of $V^{\prime}$, there is a positive integer $n_{0}$ such that $F \subset V_{n}^{\prime}$ for $n \geqq n_{0}$. Let $n \geqq n_{0}$. For every component $C$ of $V^{\prime} \backslash V_{n}^{\prime}$ 
there is $i \in\{1, \ldots, k\}$ such that $C \subset U_{p_{i}}$. Let $B_{1}, \ldots, B_{m}$ be the components of $\partial_{W} V_{n}^{\prime} \cap V^{\prime}$. Since each $f\left(B_{j}\right)$ is contained in some $\overline{f\left(U_{p_{i}} \cap V^{\prime}\right)}$, the winding number of $f\left(B_{j}\right)$ with respect to $z_{0}$ is 0 for each $j$. It follows from the argument principle that $v_{f \mid V^{\prime}}\left(z_{0}\right)=i\left(z_{0} ; f\left(\partial_{W} V^{\prime}\right)\right)$.

We will extend the notion of local degree to the generalized antecedents. So fix $p_{0} \in \beta_{V}$, and let $z_{0} \in \mathrm{Cl}\left(f ; p_{0}\right)$. Let $V^{\prime}$ be a subend of $V$ with $p_{0} \in \beta_{V^{\prime}}$ such that $\partial_{W} V^{\prime} \subset V$ and $z_{0} \notin f\left(\partial_{W} V^{\prime}\right)$. Since every neighborhood of $z_{0}$ contains points $z$ from $C \backslash \mathrm{Cl}\left(f ; \beta_{V}\right)$ with $f^{-1}(z) \cap V^{\prime} \neq \emptyset, i\left(z_{0} ; f\left(\partial_{W} V^{\prime}\right)\right)>0$ by the preceding lemma. It also appears from Lemma 2 that $i\left(z_{0} ; f\left(\partial_{W} V^{\prime}\right)\right) \geqq i\left(z_{0} ; f\left(\partial_{W} V^{\prime \prime}\right)\right)$ whenever $V^{\prime \prime} \subset V^{\prime}$. Thus, it is reasonable to set

$$
n\left(p_{0}, z_{0} ; f\right)=\inf \left\{i\left(z_{0} ; f\left(\partial_{W} V^{\prime}\right)\right)\right\},
$$

where $V^{\prime}$ runs over all subends of $V$ with $p_{0} \in \beta_{V^{\prime}}$ and $z_{0} \notin f\left(\partial_{W} V^{\prime}\right)$. It is clear that $n\left(p_{0}, z_{0} ; f\right)>0$. Further, the definition gives rise to the formula

$$
\sum_{p \in f^{-1}(z) \cap V^{\prime}} n(p ; f)+\sum_{\substack{p \in \beta_{V^{\prime}} \in \mathrm{Cl}^{\prime}(p) \\ \text { (n) }}} n(p, z ; f)=i\left(z ; f\left(\partial_{W} V^{\prime}\right)\right)
$$

for every $z \in C \backslash f\left(\partial_{W} V^{\prime}\right)$.

Remark. We point out a consequence for future use: Let $p_{0} \in \beta_{V}$, let $V^{\prime}$ be a subend of $V$ with $p_{0} \in \beta_{V^{\prime}}$, and let $z_{0} \in \mathrm{Cl}\left(f ; p_{0}\right)$. Then there is an open neighborhood $U_{z_{0}} \subset \boldsymbol{C}$ of $z_{0}$ such that $v_{f \mid V^{\prime}}(z) \geqq n\left(p_{0}, z_{0} ; f\right)$ for every $z \in U_{z_{0}} \backslash \mathrm{Cl}\left(f ; \beta_{V^{\prime}}\right)$.

3.2. We are going to show, roughly speaking, that meromorphic functions with meager cluster sets on an admissible end admit continuous extension to the ideal boundary. Besides, we obtain a condition for removability of the ideal boundary.

Theorem 8. Let $W$ be an open Riemann surface, and let $V$ be an admissible end of $W$. Suppose $f$ is a nonconstant meromorphic function on $V$ such that $\mathrm{Cl}\left(f ; \beta_{V}\right)$ is of class $N_{C}$ and no $\mathrm{Cl}(f ; p), p \in \beta_{V}$, sepurates the plane. Then $f$ admits a continuous extension to $\beta_{V}$; a fortiori, $f^{*}\left(\beta_{V}\right)$ belongs to $N_{C}^{\prime}$. Accordingly, $\beta_{V}$ is removable.

Proof. Since the problem is local and $\mathrm{Cl}\left(f ; \beta_{V}\right)$ is nowhere dense, we may assume, passing to a subend and performing an auxiliary linear fractional mapping, that $f$ is bounded on $V \cup \partial_{W} V$. Similarly, we can find a nonconstant bounded function $g$ in $M C(V)$.

As the first step, we will prove that $g^{*}\left(\beta_{V}\right)$ is totally disconnected. Let $z_{0} \epsilon$ $\mathrm{Cl}\left(f ; \beta_{V}\right)$. Modifying $V$ slightly, we obtain $z_{0} \notin f\left(\partial_{W} V\right)$. Let $m$ stand for the positive integer $i\left(z_{0} ; f\left(\partial_{W} V\right)\right)$, and denote by $G$ the component of $C \backslash f\left(\partial_{W} V\right)$ that contains $z_{0}$. Set $U=f^{-1}\left(G \backslash \mathrm{Cl}\left(f ; \beta_{V}\right)\right)$. By virtue of Lemma $2, g$ satisfies on $U$ an identity

$$
g^{m}+\sum_{i=1}^{m}\left(a_{i} \circ f\right) g^{m-i}=0,
$$

where $a_{1}, \ldots, a_{m}$ are bounded analytic functions on $G \backslash \mathrm{Cl}\left(f ; \beta_{V}\right)$ (cf. the proof of the implication $(5) \Rightarrow(4)$ in Theorem 2$)$. We proceed to show that each $a_{i}$ can be 
continued to be analytic all over $G$. So fix $z \in \mathrm{Cl}\left(f ; \beta_{V}\right) \cap G$ for a while, and let $\left(z_{n}\right)$ be a sequence of points in $G \backslash \mathrm{Cl}\left(f ; \beta_{V}\right)$ such that $z_{n} \rightarrow z$. Let $p_{1}, \ldots, p_{k}$ or $q_{1}, \ldots, q_{k^{\prime}}$ be the antecedents or the generalized antecedents of $z$, respectively. Suppose $U_{1}, \ldots, U_{k}, U_{k+1}, \ldots, U_{k+k^{\prime}}$ are mutually disjoint open neighborhoods (in $V \cup \beta_{V}$ ) of the points $p_{1}, \ldots, q_{k^{\prime}}$ such that $\partial U_{j}$ lies in $V$ for each $j$. By formula B and the ensuing remark, all antecedents of $z_{n}$ lie in $\bigcup_{j=1}^{k+k^{\prime}} U_{j}$ for large $n$. It follows that $a_{1}\left(z_{n}\right) \rightarrow$ $\sum_{j=1}^{k} n\left(p_{j} ; f\right) g\left(p_{j}\right)+\sum_{j=1}^{k^{\prime}} n\left(q_{j}, z ; f\right) g^{*}\left(q_{j}\right)$. We conclude that $a_{1}$ admits a continuous extension to $\mathrm{Cl}\left(f ; \beta_{V}\right) \cap G$. Further, by the assumption concerning $\mathrm{Cl}\left(f ; \beta_{V}\right)$, $a_{1}$ can be regarded as analytic all over $G$. Obviously, a similar reasoning applies to $a_{2}, \ldots, a_{m}$.

Denote by $\tilde{G}$ the Riemann surface of the relation

$$
P(z, w)=w^{m}+\sum_{i=1}^{m} a_{i}(z) w^{m-i}=0, \quad z \in G .
$$

Note that the number of the components of $\widetilde{G}$ is at most $m$. The mappings $c:\left(z, w_{z}\right) \mapsto z$ and $v:\left(z, w_{z}\right) \mapsto w_{z}(z)$ are analytic on $\widetilde{G}$ (cf. the proof of Theorem 2). Choose $r_{z_{0}}>0$ such that $\overline{D\left(z_{0}, r_{z_{0}}\right)} \subset G$. Then $E_{z_{0}}=v\left(c^{-1}\left(\mathrm{Cl}\left(f ; \beta_{V}\right) \cap \overline{D\left(z_{0}, r_{z_{0}}\right)}\right)\right)$ is a compact and nowhere dense subset of $\boldsymbol{C}$.

Now let $z_{0}$ vary over $\mathrm{Cl}\left(f ; \beta_{V}\right)$. From the open covering $\left\{D\left(z, r_{z}\right) \mid z \in\right.$ $\left.\mathrm{Cl}\left(f ; \beta_{V}\right)\right\}$ of $\mathrm{Cl}\left(f ; \beta_{V}\right)$ pick out a finite subcovering $\left\{D\left(z_{1}, r_{z_{1}}\right), \ldots, D\left(z_{s}, r_{z_{s}}\right)\right\}$. We will complete the first part of the proof by showing that $g^{*}\left(\beta_{V}\right) \subset \bigcup_{i=1}^{s} E_{z_{i}}$. So let $p \in \beta_{V}$, and choose a sequence of points $\left(p_{n}\right)$ in $V \backslash f^{-1}\left(\mathrm{Cl}\left(f ; \beta_{V}\right)\right)$ such that $p_{n} \rightarrow p$. We may assume, passing to a subsequence, that $f\left(p_{n}\right) \rightarrow z \in \mathrm{Cl}(f ; p)$. Now $z \in D\left(z_{i}, r_{z_{i}}\right)$ for some $i \in\{1, \ldots, s\}$. It is clear that $\left(f\left(p_{n}\right), g\left(p_{n}\right)\right)$ satisfies a relation of type (1), say $P\left(f\left(p_{n}\right), g\left(p_{n}\right)\right)=0$, for large $n$. By continuity, the same holds for $\left(z, g^{*}(p)\right)$, i.e., $P\left(z, g^{*}(p)\right)=0$. But this implies that $g^{*}(p) \in v\left(c^{-1}(z)\right)$. Hence $g^{*}(p) \in E_{z_{i}}$. Thus $g^{*}\left(\beta_{V}\right) \subset \bigcup_{i=1}^{s} E_{z_{i}}$. So by Theorem $1, g^{*}\left(\beta_{V}\right)$ is totally disconnected as was asserted. We note that the removability of $\beta_{V}$ could now be readily established by observing that $E_{z_{i}} \in N_{C}$ for each $i$. Of course, this also follows from the claim we are going to prove next: that $\mathrm{Cl}\left(f ; \beta_{V}\right)$ is totally disconnected.

The set $g^{*}\left(\beta_{V}\right)$ being totally disconnected, it obviously suffices to prove that $\mathrm{Cl}\left(f ; \beta_{V}\right) \cap \overline{D\left(z_{0}, r_{z_{i}}\right)} \subset c\left(v^{-1}\left(g^{*}\left(\beta_{V}\right) \cap E_{z_{i}}\right)\right)$ for each $i \in\{1, \ldots, s\}$; of course, $c$ and $v$ here stand for the center mapping and the value mapping associated with the point $z_{i}$. So fix $i \in\{1, \ldots, s\}$, and let $z \in \mathrm{Cl}\left(f ; \beta_{V}\right) \cap \overline{D\left(z_{i}, r_{z_{i}}\right)}$. Pick out a point $p \in \beta_{V}$ such that $z \in \mathrm{Cl}(f ; p)$. Then we can find a sequence of points $\left(p_{n}\right)$ in $V$ such that $p_{n} \rightarrow p$ and $f\left(p_{n}\right) \rightarrow z$. Clearly, we may also assume that $f\left(p_{n}\right) \notin \mathrm{Cl}\left(f ; \beta_{V}\right)$ for all $n$. Now, $\left(f\left(p_{n}\right), g\left(p_{n}\right)\right)$ satisfies a relation of type $(1)$, say $P\left(f\left(p_{n}\right), g\left(p_{n}\right)\right)=0$, for large $n$. By continuity, the same is true of $\left(z, g^{*}(p)\right)$. Hence there is a function element $w_{z}$ with center $z$ associated with the relation $P=0$ such that $v\left(z, w_{z}\right)=g^{*}(p)$. But this means that $z \in c\left(v^{-1}\left(g^{*}(p)\right) \subset c\left(v^{-1}\left(g^{*}\left(\beta_{V}\right) \cap E_{z_{i}}\right)\right)\right.$, whence the claim follows. Now, by conncetedness, each $\mathrm{Cl}(f ; p)$ reduces to a singleton. Therefore $f$ extends to a continuous mapping of $V \cup \beta_{V}$. 
Corollary 1. Let $W$ be an open Riemann surface, and let $E$ be a closed, totally disconnected subset of $W$. Suppose $f$ is a meromorphic function on $W \backslash E$ such that the closed parts of $\mathrm{Cl}(f ; E)$ are of class $N_{C}$ and no $\mathrm{Cl}(f ; p), p \in E$, separates the plane. Then $f$ can be continued to be meromorphic on $W$.

Proof. Fix $p \in E$, and choose a relatively compact region $V$ in $W$ such that $p \in V$ and $E \cap \partial V=\emptyset$. Certainly $V \backslash E$ is then an admissible end of $W \backslash E$. It follows from the preceding theorem, in view of the remarks following Theorem 5 , that $f$ can be extended to be meromorphic in a neighborhood of $p$. Since $p$ was arbitrary, the proof is complete.

The next result is due to Ishchanov [5].

Corollary 2. Let $G$ be a plane domain, and let $E$ be a closed totally disconnected subset of $G$. Suppose $f$ is an analytic function on $G \backslash E$ such that $\operatorname{Re} f$ admits a continuous extension to $E$ and takes a constant value there. Then $f$ can be extended to be analytic on $G$.

Proof. By assumption, we have a continuous function $h$ on $G$ and a constant $c$ with $h \mid G \backslash E=\operatorname{Re} f$ and $h(p)=c$ for all $p \in E$. Plainly, this implies $\mathrm{Cl}(f ; p) \subset L=$ $\{z \in \mathbb{C} \mid \operatorname{Re} z=c\} \cup\{\infty\}$ for each $p \in E$.

Fix $p_{0} \in E$ and let $G^{\prime} \subset G$ be a Jordan domain with $p_{0} \in G^{\prime}, \partial G^{\prime} \subset G$ analytic and $\partial G^{\prime} \cap E=\emptyset$. Pick out a finite point $z_{0} \in L \backslash f\left(\partial G^{\prime}\right)$, and choose $r>0$ such that $D\left(z_{0}, r\right) \cap f\left(\partial G^{\prime}\right)=\emptyset$. Then $v_{f \mid G^{\prime} \backslash E}$ is finite and constant, say $n^{\prime}$, in $D\left(z_{0}, r\right) \cap$ $\{z \in C \mid \operatorname{Re} z<c\}$; similarly, there is a nonnegative integer $n^{\prime \prime}$ such that $v_{f \mid G^{\prime} \backslash E}(z)=n^{\prime \prime}$ for $z \in D\left(z_{0}, r\right) \cap\{z \in C \mid \operatorname{Re} z>c\}$. It is now readily seen that there are at most $n^{\prime}+n^{\prime \prime}$ points $p_{i}, i=1, \ldots, k$, in $E \cap G^{\prime}$ such that $z_{0} \in \mathrm{Cl}\left(f ; p_{i}\right)$. Thus for each $p \in E \cap G^{\prime} \backslash$ $\left\{p_{1}, \ldots, p_{k}\right\} \mathrm{Cl}(f ; p)$ is a proper subset of $L$. By Corollary $1, f$ admits a meromorphic extension $f^{*}$ to $G^{\prime} \backslash\left\{p_{1}, \ldots, p_{k}\right\}$. But $p_{i}, i=1, \ldots, k$, being now isolated singularities, $f^{*}$ can be taken as meromorphic all over $G^{\prime}$. Since the arising of poles evidently contradicts the hypothesis of the corollary, the assertion is proved.

\section{References}

[1] Ahlfors, L., and A. Beurling: Conformal invariants and function-theoretic null-sets. - Acta Math. 83, 1950, 101-129.

[2] Church, P. T., and E. Hemmingsen: Light open maps on $n$-manifolds. - Duke Math. J. 27, 1960, 527-536.

[3] Garnett, J.: Analytic capacity and measure. - Lecture Notes in Mathematics 297, SpringerVerlag, Berlin-Heidelberg-New York, 1972.

[4] Heins, M.: Riemann surfaces of infinite genus. - Ann. of Math. 55, 1952, 296-317.

[5] Ishchanov, B. Zh.: On a theorem of V. S. Fedorov. - Moscow Univ. Math. Bull. 36:3, 1981, $52-56$. 
[6] JÄRVI, P.: Meromorphic functions on certain Riemann surfaces with small boundary. - Ann. Acad. Sci. Fenn. Ser. A I Math. 5, 1980, 301-315.

[7] JÄrVI, P.: On certain algebras of analytic functions on Riemann surfaces. - Ann. Acad. Sci. Fenn. Ser. A I Math. 5, 1980, 317-326.

[8] JärvI, P.: A note on maximum modulus algebras. - Ann. Acad. Sci. Fenn. Ser. A I Math. 6, 1981, 173-178.

[9] Myrberg, L.: Über quasirationale Funktionen auf parabolischen Riemannschen Flächen. Ann. Acad. Sci. Fenn. Ser. A I Math. 404, 1967, 1-33.

[10] Ozawa, M.: Meromorphic functions on certain Riemann surfaces. - Proc. Amer. Math. Soc. 16, 1965, 747-751.

[11] Richards, I.: On the classification of noncompact surfaces. - Trans. Amer. Math. Soc. 106, 1963, 259-269.

[12] Royden, H. L.: Riemann surfaces with the absolute AB-maximum principle. - Proceedings of the Conference on Complex Analysis, Minneapolis, 1964, edited by A. Aeppli, E. Calabi and H. Röhrl, Springer-Verlag, Berlin-Heidelberg-New York, 1965, 172-175.

[13] Royden, H. L.: Algebras of bounded analytic functions on Riemann surfaces. - Acta Math. $114,1965,113-142$.

[14] Rudin, W.: Some theorems on bounded analytic functions. - Trans. Amer. Math. Soc. 78, 1955, 333-342.

[15] Sario, L., and M. NAKaI: Classification theory of Riemann surfaces. - Die Grundlehren der mathematischen Wissenschaften 164, Springer-Verlag, Berlin-Heidelberg-New York, 1970.

[16] SARIo, L., and K. OiKawa: Capacity functions. - Die Grundlehren der mathematischen Wissenschaften 149, Springer-Verlag, Berlin-Heidelberg-New York, 1969.

[17] Stoïlow, S.: Leçons sur les principes topologiques de la théorie des fonctions analytiques. Gauthier-Villars, Paris, 1956.

[18] Titus, C. J., and G. S. Young: The extension of interiority, with some applications. - Trans. Amer. Math. Soc. 103, 1962, 329-340.

[19] Wermer, J.: The maximum principle for bounded functions. - Ann. of Math. 69, 1959, 598604.

[20] Whyburn, G. T.: Topological analysis. - Princeton University Press, Princeton, New Jersey, 1958.

University of Helsinki

Department of Mathematics

SF-00100 Helsinki 10

Finland

Received 21 September 1983 